\title{
Incidence of cardiovascular disease in Type 1 (insulin-dependent) diabetic subjects with and without diabetic nephropathy in Finland
}

\author{
J. Tuomilehto ${ }^{1}$, K. Borch-Johnsen ${ }^{2}$, A. Molarius ${ }^{1}$, T. Forsén ${ }^{1}$, D. Rastenyte ${ }^{1}$, C. Sarti ${ }^{1}$, A. Reunanen ${ }^{3}$ \\ ${ }^{1}$ Diabetes and Genetic Epidemiology Unit, Department of Epidemiology and Health Promotion, \\ National Public Health Institute, Helsinki, Finland \\ ${ }^{2}$ Steno Diabetes Centre, Gentofte, Denmark \\ ${ }^{3}$ National Public Health Institute, Department of Health and Disability, Helsinki, Finland
}

\begin{abstract}
Summary This study evaluates the impact of diabetic nephropathy on the incidence of coronary heart disease, stroke and any cardiovascular disease in the Finnish population, which has a high risk of Type 1 (insulindependent) diabetes mellitus and cardiovascular disease. We performed a prospective analysis of the incidence of coronary heart disease, stroke and cardiovascular disease in all Type 1 subjects in the Finnish Type I diabetes mellitus register diagnosed before the age of 18 years between 1 January 1965 and 31 December 1979 nationwide. The effect of age at onset of diabetes, attained age at the end of follow-up, sex, diabetes duration and of the presence of diabetic nephropathy on the risk for cardiovascular disease was evaluated. Cases of nephropathy, coronary heart disease, stroke and all cardiovascular diseases were ascertained from the nationwide Finnish Hospital Discharge Register and National Death Register using computer linkage with the Type I diabetes mellitus register. Of the 5148 Type 1 diabetic patients followed up, 159 had a cardiovascular
\end{abstract}

event of which 58 were coronary heart diseases, 57 stroke events and 44 other heart diseases. There were virtually no cases of cardiovascular disease before 12 years diabetes duration. The cumulative incidence of cardiovascular disease by the age of 40 years was $43 \%$ in Type 1 diabetic patients with diabetic nephropathy, compared with $7 \%$ in patients without diabetic nephropathy, similarly in men and women. The relative risk for Type 1 diabetic patients with diabetic nephropathy compared with patients without diabetic nephropathy was 10.3 for coronary heart disease, 10.9 for stroke and 10.0 for any cardiovascular disease, similarly in men and women. The presence of nephropathy in Type I diabetic subjects increases not only the risk of coronary heart disease, but also of stroke by tenfold. [Diabetologia (1998) 41: 784-790]

Keywords Diabetes mellitus, nephropathy, cardiovascular disease, epidemiology, stroke, coronary heart disease.
When insulin became available in the $1920 \mathrm{~s}$, a major shift occurred in the distribution of causes of death in Type 1 (insulin-dependent) diabetic patients away

Received: 14 August 1997 and in final revised form: 2 March 1998

Corresponding author: Professor J. Tuomilehto, Diabetes and Genetic Epidemiology Unit, Department of Epidemiology and Health Promotion, National Public Health Institute, Mannerheimintie 166, FIN - 00300 Helsinki, Finland

Abbreviations: Cardiovascular disease, CVD; coronary heart disease, CHD; confidence interval, CI; diabetic nephropathy, DN; Hospital Discharge Register, HDR; personal identification code, ID; acute myocardial infarction, AMI. from diabetic coma, which dominated in the pre-insulin era, to renal and cardiac diseases [1]. At present, renal disease is the leading cause of death during the early years of Type 1 diabetes, while after 30 years of diabetes two-thirds of the deaths are due to cardiovascular disease (CVD) [2,3]. A strong link exists between diabetic nephropathy (DN) and death from CVD in Type 1 diabetic patients [4]. Mortality [5-7] and morbidity from CVD [8] are much higher in patients with proteinuria than without proteinuria, and a common pathogenic mechanism for diabetic microangiopathy and macroangiopathy has been suggested [9].

To our knowledge, all but two of the previous studies $[8,10]$ have only assessed mortality from CVD, 
while the incidence of non-fatal CVD in Type I diabetic patients has not been shown. Since only onethird of acute coronary and stroke events are fatal $[11,12]$, studies on CVD in Type 1 diabetic patients including fatal CVD alone do not give a complete picture of the risk of CVD in the study population. The two studies $[8,10]$ both reported an increased incidence of coronary heart disease (CHD) in patients with DN compared with patients without DN, but these were case-control studies including a limited number of subjects and their results need to be confirmed in a larger population sample.

Finland has the highest incidence of Type 1 diabetes in the world [13], and although some data about the late complications in this country exist [14], more studies are needed. The incidence and mortality rates in Finland of the main forms of CVD, i.e. CHD and stroke, are also known to be among the highest in the world, although steeply declining trends in both CHD [15] and stroke [12] mortality have recently been reported. The aim of the present study was to analyse the risk of CVD in a large and representative cohort of young-onset Type 1 diabetic patients. Furthermore, we assessed the link between diabetic nephropathy and CVD in this population at high risk both for Type 1 diabetes and CVD.

\section{Subjects, materials and methods}

The Finnish IDDM register, which is population-based, was used to identify all Finnish Type I diabetic patients diagnosed before the age of 18 years from 1 January 1965 to 31 December 1979. This register is based on the Social Insurance Institution Central Drug Register which was started in 1964 and contains information on all patients receiving state-subsidized medication for chronic disorders. These subjects can be identified through the unique personal identification (ID) code (containing information on date of birth, sex and control-digits). Insulin treatment prescribed by a physician is free of charge for diabetic patients in Finland. All Type 1 diabetic patients applying for free insulin are entered in the Type I diabetes mellitus register. The nationwide Finnish Type I diabetes mellitus database of cases diagnosed from 1965 to 1979 has been described in detail previously [16-18], and the coverage is well above $95 \%$ [19]. Patients diagnosed during 1965 to 1969 who died before the end of 1969 were not included in the study cohort.

A total of 5148 IDDM patients diagnosed at 17 years of age or younger fulfilled the inclusion criteria for the Finnish Type I diabetic registry. Using the ID-number the cohort was followed-up for death or hospitalization with a diagnosis of $\mathrm{DN}$, CHD, stroke, or any CVD through record linkage with the nationwide Hospital Discharge Register (HDR). The HDR covers in-patient discharges from all hospitals nationwide after 1968 and includes the ID code of the patient, dates of admission and discharge for each hospitalization, and up to four discharge diagnoses. Since the HDR data collected during the first 2 years were incomplete [20], we included hospitalizations starting from 1 January 1970 to 31 December 1989 in the present analyses. Mistakes in the ID codes in HDR were found, leading to unsuccessful record linkage in about $5 \%$ of the cases diagnosed before 1985 [20, 21]. In 1986, when computer logical checks were introduced, the proportion of unsuccessful record linkage fell to $0.3 \%$ [20]. The attained age at the end of the follow-up ranged from 10 years to 42 years, and the mean age attained was 26.7 years.

For record linkage we based the identification of diabetic nephropathy on hospitalizations with a discharge diagnosis of diabetic renal disease. A similar procedure was used for CVD. Non-fatal acute CVD events not leading to hospitalization could be ascertained only if the patient was later hospitalized with a CVD diagnosis; fatal cases were fully covered. It has been shown that patients below the age of 65 years suffering from acute CHD or stroke are almost always hospitalized. In Finland, a Nordic variant of the 8th version of the International Classification of Diseases (ICD) (adding a fifth digit after the usual 4 digits given to each disease) was used from 1968 to 1986 . From 1987 onwards, the Nordic variant of 9th revision was adopted. In ICD 8 the code 250.04 and in ICD 9 the code 2503 was used to identify cases of nephropathy. The first three digits of the ICD code in both ICD 8 and ICD 9 were used for identifying CHD: 410-414, stroke: 430-438, CVD (CHD, stroke and other heart diseases): 410-414, 420-429, 430-438. Thus CVD refers to the broader category of cardiovascular and cerebrovascular diseases. Rheumatic heart disease as well as atherosclerotic and other diseases were not included, both because the cohort was still relatively young at the end of follow-up, and because they represent mostly chronic and unspecified disorders. The date of diagnosis was, by definition, the admission date of the first hospitalization with one of the above-mentioned diagnoses. In Finland, the treating physician assigns all clinical diagnoses on admission and discharge, including the ICD codes appearing in the HDR.

A few patients $(n=18)$ died of CVD without being hospitalized. Therefore these cases were only found in the National Death Register and not in the HDR. The date of death was used as the date of diagnosis of the CVD events in these patients. Fatal cases of CHD were defined as having died within 30 days of the first diagnosis of CHD.

For simplicity, we refer in the text to "diabetic nephropathy (DN)" when talking about "first hospitalization for diabetic nephropathy". Accordingly, "coronary heart disease", "stroke", and "all CVD" should thus be read as "first hospitalization for acute coronary heart disease", "first hospitalization for acute stroke", and "first hospitalization for any CVD", respectively.

The cumulative incidence, life-table and Cox regression analyses were produced with SAS software (Cary, NC, USA). The cumulative incidence was calculated according to the lifetable method using the SAS LIFETEST procedure, with nephropathy as a categorical variable. This means that a subject who has developed nephropathy belongs to the DN group regardless of the date of onset of nephropathy. A $p$-value below 0.05 was considered statistically significant. In the Cox regression models presented in Tables 3 and 4, where we explored the effect of the age at onset of Type 1 diabetes on the risk of CHD, stroke, and all CVD, the patients with onset between 0-4 years were used as the reference group. As age, age at onset of diabetes and diabetes duration are direct functions of each other, it is not possible to introduce age at onset of Type 1 diabetes, duration of diabetes and attained age at the end of the follow-up in the same model. We thus made two separate models, one including age at Type 1 diabetes onset with the duration of diabetes as covariate, and another including age at onset with attained age as covariate. In the Cox model in Table 2 , the patients with nephropathy were compared with patients without nephropathy (reference group). Unlike in the life-table analyses, in the Cox models nephropathy was modeled as a time-dependent variable. Therefore patients who de- 
Table 1. Number of cases of CHD, stroke and all CVD during the follow-up from 1975 to 1989 in subjects in the Finnish Type I diabetes mellitus register with (DN) and without (non$\mathrm{DN})$ diabetic nephropathy

\begin{tabular}{lllll}
\hline & & $\begin{array}{l}\text { DN } \\
(n=446)\end{array}$ & $\begin{array}{l}\text { Non-DN } \\
(n=4702)\end{array}$ & $\begin{array}{l}\text { All } \\
(n=5148)\end{array}$ \\
\hline CHD & Men & 17 & 18 & 35 \\
& Women & 10 & 13 & 23 \\
\multirow{4}{*}{ Stroke } & Both & 27 & 31 & 58 \\
& Men & 13 & 14 & 27 \\
& Women & 12 & 18 & 30 \\
All CVD & Both & 25 & 32 & 57 \\
& Men & 38 & 53 & 91 \\
& Women & 26 & 42 & 68 \\
& Both & 64 & 95 & 159 \\
\hline
\end{tabular}

veloped DN were included in the non-nephropathic group until the diagnosis of DN had been made, as described previously by Borch-Johnsen et al. [6].

\section{Results}

Of the 5148 patients, 159 were diagnosed as having had CVD during the follow-up. Of these, 58 had suffered from CHD and 57 from stroke and 44 from other heart diseases (Table 1 ). Type 1 diabetic patients suffering from CHD, stroke or any CVD were on average slightly older at diagnosis of these CVD events (29.0) in the DN group than in the non-DN group (26.5).

The cumulative incidence of CVD by the age of 40 years was $43 \%$ in Type 1 diabetic subjects with $\mathrm{DN}$ and only $7 \%$ in subjects without DN. The respective cumulative incidences of CHD and stroke by the age of 40 were $13 \%$ and $20 \%$ in diabetic patients with DN but only $4 \%$ and $2 \%$ in patients without DN (Fig. 1).

In patients with $\mathrm{DN}$, cardiovascular complications occurred at approximately $12-13$ years after the diagnosis of diabetes, whereas in subjects without DN the lag time was slightly longer, $16-17$ years (Fig. 2). The incidence of CHD and stroke increased linearly, both reaching approximately $11 \%$ after 24 years duration of diabetes. The cumulative incidence of CVD after 24 years of diabetes was $24 \%$ in this cohort.

The incidence of CVD was clearly related to the duration of nephropathy (Fig. 3), the trend being $2.3 \%$ per year after the diagnosis of nephropathy. In patients with nephropathy there was a linear increase of $1.6 \%$ per year in the cumulative incidence of $\mathrm{CHD}$, which was even steeper than that of stroke $(0.8 \%$ per year $)$, starting at the time of diagnosis of nephropathy. Applying the Cox regression model we found that the risk of getting CHD, stroke or any CVD, was approximately 10 times higher in DN
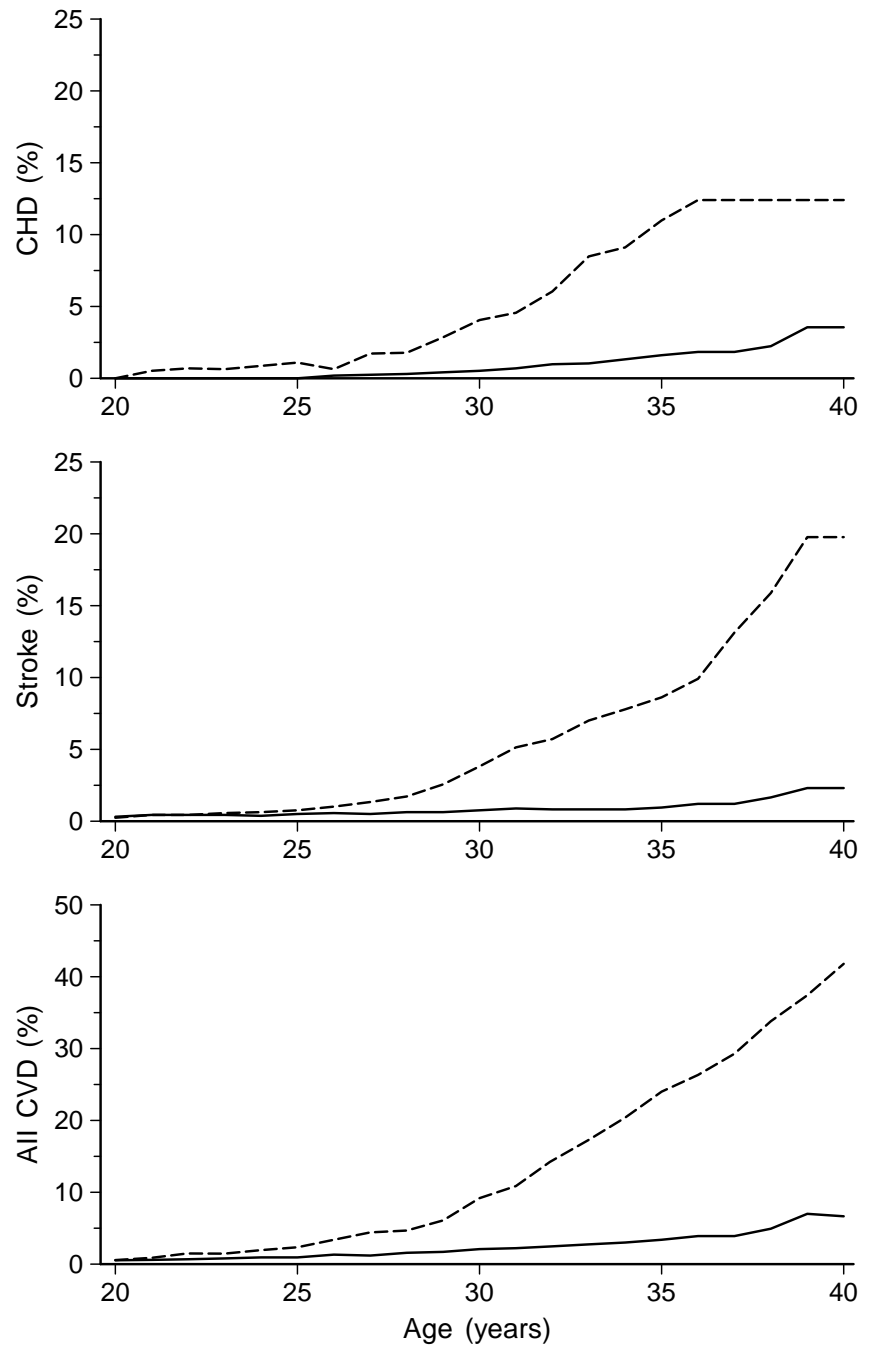

Fig. 1. Cumulative incidence of CHD, stroke and all CVD in patients with Type I diabetic subjects with $(n=4702)(---)$ and without $(-)$ diabetic nephropathy $(n=446)$, by age

than non-DN patients (Table 2). The impact of DN on fatal CHD (relative hazard rate: $10.6,95 \% \mathrm{CI}$ : 3.5-32.4) was comparable with that on non-fatal acute CHD events (relative hazard rate: 9.7, 95\% CI: 6.3-15.0).

No differences were found between men and women in the cumulative incidence of CVD concerning duration of diabetes (Table 3 ) or the age attained at the end of follow-up (Table 4). Also the age at onset of Type 1 diabetes did not seem to influence the risk of cardiovascular disease (Tables 3 and 4). In fact, the apparently higher cardiovascular risk in subjects with later onset of diabetes (Table 3 ) was totally reversed when the attained age at the end of followup was introduced in the model (Table 4). This is due to the shorter duration of diabetes in patients diagnosed at an older age. The risk of CHD, stroke, and all CVD imparted by DN in these larger models did not substantially change, remaining over 9 times higher in DN compared with non-DN subjects. 

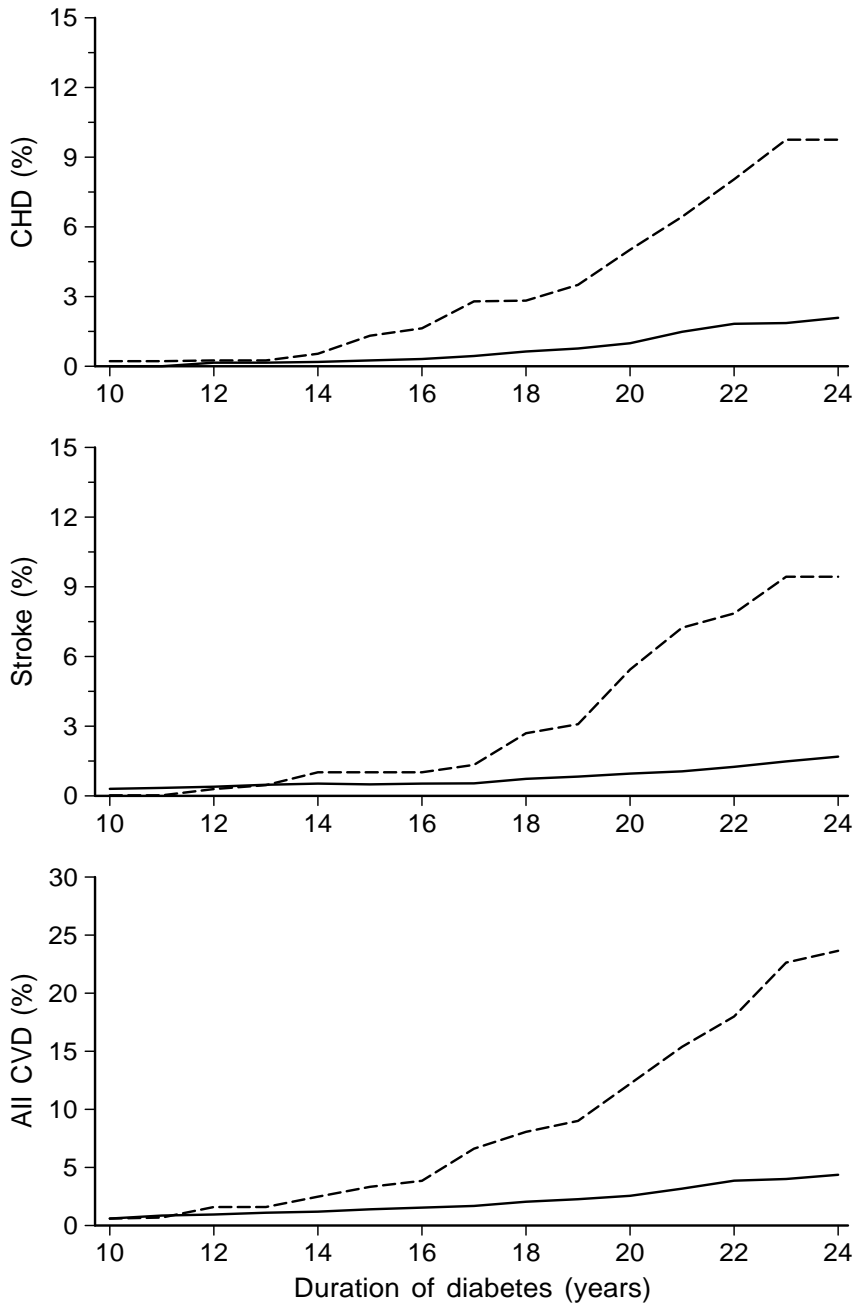

Fig. 2. Cumulative incidence of CHD, stroke and all CVD in patients with Type I diabetic subjects with $(n=4702)(---)$ and without $(-)$ diabetic nephropathy $(n=446)$, by duration of diabetes

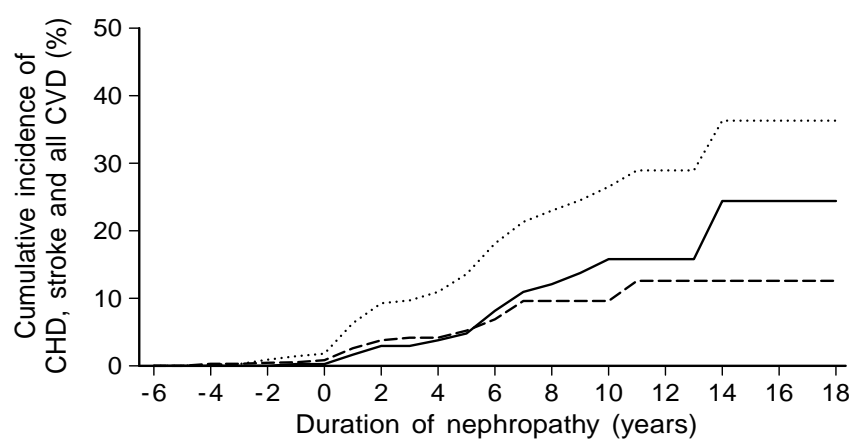

Fig. 3. Cumulative incidence of CHD (-), stroke (---) and any CVD $(\ldots$.$) by the duration of diabetic nephropathy$ $(n=446)$

\section{Discussion}

In our large population-based cohort we found that the risk of developing CHD, stroke or any CVD is 10 times increased in the Type 1 diabetic patients with
Table 2. Relative hazard rates for the risk of developing CHD, stroke or any CVD in Type 1 diabetic subjects with diabetic nephropathy $(n=446)$ compared with Type 1 diabetic subjects without nephropathy $(n=4702)$ during the 10-24-year followup

\begin{tabular}{lclll}
\hline & $\begin{array}{l}\text { Number } \\
\text { of cases }\end{array}$ & $\begin{array}{l}\text { Relative } \\
\text { hazard rate }\end{array}$ & $95 \%$ CI & $p$-value \\
\hline CHD & 58 & 10.3 & $6.0-17.7$ & 0.0001 \\
Stroke & 57 & 10.9 & $6.0-19.8$ & 0.0001 \\
CVD & 159 & 10.3 & $7.0-14.1$ & 0.0001 \\
\hline
\end{tabular}

Nephropathy was entered in the Cox-regression model as a time-dependent variable

Table 3. Relative hazard rates of nephropathy, sex, and age at onset of Type 1 diabetes for CHD, stroke, and all CVD in all diabetic subjects, by duration of diabetes

\begin{tabular}{llll}
\hline Risk factor & $\begin{array}{l}\text { CHD } \\
\text { Relative } \\
\text { hazard rate }\end{array}$ & $\begin{array}{l}\text { Stroke } \\
\text { Relative } \\
\text { hazard rate }\end{array}$ & $\begin{array}{l}\text { All CVD } \\
\text { Relative } \\
\text { hazard rate }\end{array}$ \\
\hline Nephropathy & $8.2^{\mathrm{d}}$ & $6.4^{\mathrm{d}}$ & $7.7^{\mathrm{d}}$ \\
Gender & 0.7 & 1.2 & 0.9 \\
Onset at 5-9 years & 3.2 & 1.3 & 1.6 \\
Onset at 10-14 years $^{\mathrm{a}}$ & $5.0^{\mathrm{b}}$ & 1.6 & $2.6^{\mathrm{c}}$ \\
Onset at 15-17 years $^{\mathrm{a}}$ & $6.3^{\mathrm{c}}$ & 1.6 & $3.1^{\mathrm{d}}$ \\
\hline
\end{tabular}

Nephropathy was entered in the Cox-regression model as time-dependent variable.

${ }^{a}$ Compared with those whose onset of Type I diabetes was at $0-4$ years of age; ${ }^{\mathrm{b}} p<0.05 ;{ }^{\mathrm{c}} p<0.01 ;{ }^{\mathrm{d}} p<0.001$

Table 4. Relative hazard rates of nephropathy, sex, and age at onset of Type I diabetes for CHD, stroke, and all CVD in Type I diabetes subjects, by attained age at the end of the follow-up period

\begin{tabular}{llll}
\hline Risk factor & $\begin{array}{l}\text { CHD } \\
\text { Relative } \\
\text { hazard rate }\end{array}$ & $\begin{array}{l}\text { Stroke } \\
\text { Relative } \\
\text { hazard rate }\end{array}$ & $\begin{array}{l}\text { All CVD } \\
\text { Relative } \\
\text { hazard rate }\end{array}$ \\
\hline Nephropathy & $9.1^{\mathrm{c}}$ & $9.0^{\mathrm{c}}$ & $9.3^{\mathrm{c}}$ \\
Gender & 0.8 & 1.3 & 0.9 \\
Onset at 5-9 years & 1.0 & 0.6 & 0.8 \\
Onset at 10-14 years $^{\mathrm{a}}$ & 0.6 & 0.4 & 0.7 \\
Onset at 15-17 years $^{\mathrm{a}}$ & 0.4 & $0.2^{\mathrm{b}}$ & 0.5 \\
\hline
\end{tabular}

Nephropathy was entered in the Cox-regression model as time-dependent variable.

${ }^{a}$ Compared with those whose onset of Type I diabetes was at $0-4$ years of age; ${ }^{\mathrm{b}} p<0.01 ;{ }^{\mathrm{c}} p<0.001$

DN compared with those without DN. We did not observe any differences in the risk of CVD between men and women. The risk of both nephropathy and CVD increases with diabetes duration. Our results are in keeping with findings from previous studies [4-7]. Lounamaa [14] compared the risk of fatal CVD among Type 1 diabetic patients with the general population in Finland. The excess mortality due to circulatory diseases was 126-fold among females and 58-fold among males with DN when compared with the general population. In the Type 1 diabetic patients without DN the excess mortality was considerably lower, in females 3.8-fold and males 4.5-fold when compared 
with the general population [14]. Non-fatal CVD events were not included in that analysis.

The present study was based on record linkage of routinely collected data. Analyses are based on hospital discharge diagnoses, leaving the possibility of misclassification of patients with respect to DN as well as CVD. The number of false positive DN in our diabetic patients should be very small, since our study included hospitalized cases only. Unfortunately, no study in Finland has thus far validated the prevalence of DN among hospitalized compared to nonhospitalized Type 1 diabetic subjects. Assuming that the non-hospitalized DN subjects have the same risk of developing CVD as hospitalized subjects, this would underestimate the real risk of CVD in DN, which we found to be approximately 10 -fold. In addition, it is unlikely that the incidence of DN was grossly underestimated in our study. If it were, one would expect that an excess of DN cases are diagnosed during the hospitalization for CVD. This was, however, not the case in our cohort, since no peak in CVD incidence at the onset of DN was seen (Fig. 3). The incidence of non-fatal cardiovascular and cerebrovascular disease could instead be underreported, especially in subjects without $\mathrm{DN}$, since they are hospitalized less often than subjects with DN. This would in turn overestimate the risk of CVD in DN subjects. However this is unlikely to produce a significant bias, since the validity of the acute myocardial infarction (AMI) diagnoses in the HDR is good and has been found to be adequate for both epidemiological and clinical studies [20]. The incidence of hospitalization for DN is comparable to other European countries and has not changed during the last decades [22].

In the FINMONICA AMI register, between 1983 and 1992, the proportion of non-hospitalized myocardial infarctions (including definite and possible myocardial infarctions) in the age group 35-64 years was between 21 and $34 \%$ in men and 12 and $18 \%$ in women [23]. Even admitting a similar (although improbable, given that in the age range of our population the prevalence of CVD is much lower) range of underestimation of cases in our Type 1 diabetic subjects without DN, the risk of CVD would remain substantially lower in this group compared with diabetic subjects with DN. In the age group 25-44 years, the incidence of AMI was 99 [11] and of stroke 57 [24] per 100000 individuals.

There were no substantial differences in the incidence of cardiovascular and cerebrovascular disease in diabetic men and women. This is in accordance with previously observed data [7], but different from the general population, where the incidence of AMI and stroke is higher in men $[11,12]$ than women. It has been suggested $[3,5,6]$ that low age at onset is a risk factor for micro- and macrovascular complications. This is controversial and difficult to analyse. In our cohort, the risk of CHD, stroke, or any CVD de- pended substantially on the presence or absence of $\mathrm{DN}$ and on the duration of diabetes, but not on the age at onset of diabetes. The risk of any of these cardiovascular events was similar in men and women, which is in keeping with the previous observation that the female advantage regarding CVD is lost in diabetic patients [25].

The risk of stroke among patients with DN was 10 times higher than among those without DN, an increase similar to that observed for CHD. Type 2 (non-insulin-dependent) diabetes is an established risk factor for stroke, and in a previous study we found a sixfold increased risk of dying from cerebrovascular disease among adult diabetic subjects (primarily Type 2 diabetic), compared with the nondiabetic population [26]. This is, however, the first prospective population-based study showing a strong relation between Type 1 diabetes and stroke in patients aged less than 40 years.

The causes of the increased frequency of both CHD and stroke among Type 1 diabetic patients with clinical DN remains largely unknown, but it may in part be explained by differences between patients with and without DN in conventional risk factors, such as high blood pressure, lipids and lipoproteins, modified by genetic factors. Diabetic patients with nephropathy have been reported to have higher blood pressure [27-29], higher prevalence of smoking [30], increased plasma fibrinogen and increased serum total cholesterol [10, 31-33] when compared with patients without clinical nephropathy. These are all risk factors for macrovascular disease, and together with the increased transcapillary escape rate of albumin [31] (a marker of generalized increase in vascular permeability), they may explain the increased risk of macrovascular disease as also suggested by Deckert et al. [9]. Perturbations in plasma lipoproteins have been proposed as one pathogenic mechanism responsible for the increase of CHD risk in diabetic nephropathy $[8,34]$. The same mechanisms could also intervene in increased stroke risk. In contrast to normoalbuminuric Type 1 diabetic patients with good to moderate glycaemic control $[35,36]$, proteinuric diabetic patients show elevations of serum low-density lipoproteins (LDL), cholesterol and serum triglycerides, and lowering of plasma high-density lipoprotein (HDL) $[8,34,37]$. In the general population, these are also risk factors for $\mathrm{CHD}[38,39]$. The relationship between perturbation in plasma lipoproteins in diabetic subjects and the increased risk of cardiovascular and cerebrovascular disease is largely unexplained and certainly not unequivocal. In a study by Karhi et al. [37], for example, the slight decreases in serum HDL cholesterol in albuminuric groups was not sufficient to explain the excess CHD risk.

Low cardiac parasympathetic activity has been shown, both in experimental [40] and clinical studies [41] of non-diabetic patients with CHD, to be a major 
risk factor for sudden cardiac death by lowering the threshold for malignant arrhythmia [40, 42]. Damage to the cardiac autonomic system is prevalent in diabetic subjects [43], particularly in patients with nephropathy [44]. This could explain the high rate of sudden cardiac death in patients with DN.

In conclusion, this study shows that DN severely increases the risk of CHD, stroke, and all CVD. In a country such as Finland, where the occurrence of CHD and stroke is high, we observed a 10-fold increase in the risk of such diseases in Type 1 diabetic patients with DN compared with those without DN. In our study the increased risk was similar in men and women, and depended substantially on the duration of diabetes and on the presence of DN, but not on the age of onset of Type I diabetes mellitus.

\section{References}

1. Entmacher PS, Root HF, Marks HH (1964) Longevity of diabetic patients in recent years. Diabetes 13: 373-377

2. Borch-Johnsen K, Nissen H, Henriksen E et al. (1987) The natural history of insulin-dependent diabetes mellitus in Denmark: 1 . Long-term survival with and without late diabetic complications. Diabet Med 4: 201-210

3. Krolewski AS, Warram JH, Christlieb AR, Busick EJ, Kahn CR (1985) The changing natural history of nephropathy in type I diabetes. Am J Med 78: 785-794

4. Krolewski AS, Kosinski EJ, Warram JH et al. (1987) Magnitude and determinants of coronary artery disease in juvenile-onset, insulin-dependent diabetes mellitus. Am J Cardiol 59: 750-755

5. Andersen AR, Christiansen JS, Andersen JK, Kreiner S, Deckert T (1983) Diabetic nephropathy in type 1 (insulindependent) diabetes: an epidemiological study. Diabetologia 25: 496-501

6. Borch-Johnsen K, Andersen PK, Deckert T (1985) The effect of proteinuria on relative mortality in type 1 (insulindependent) diabetes mellitus. Diabetologia 28: 590-596

7. Borch-Johnsen K, Kreiner S (1987) Proteinuria: value as predictor of cardiovascular mortality in insulin dependent diabetes mellitus. BMJ 294: 1651-1654

8. Winocour PH, Durrington PN, Ishola M, Anderson DC, Cohen H (1987) Influence of proteinuria on vascular disease, blood pressure, and lipoproteins in insulin dependent diabetes mellitus. BMJ 294: 1648-1651

9. Deckert T, Feldt-Rasmussen B, Borch-Johnsen K, Jensen T, Kofoed-Enevoldsen A (1989) Albuminuria reflects widespread vascular damage. The Steno hypothesis. Diabetologia 32: 219-226

10. Jensen T, Borch-Johnsen K, Kofoed-Enevoldsen A, Deckert T (1987) Coronary heart disease in young type 1 (insulin-dependent) diabetic patients with and without diabetic nephropathy: incidence and risk factors. Diabetologia 30: 144-148

11. Tuomilehto J, Arstila M, Kaarsalo E et al. (1992) Acute myocardial infarction (AMI) in Finland - baseline data from the FINMONICA AMI register in 1983-1985 [published erratum appears in Eur Heart J 1992 13(8):1153]. Eur Heart J 13: 577-587

12. Tuomilehto J, Rastenyte D, Sivenius J et al. (1996) Tenyear trends in stroke incidence and mortality in the FINMONICA Stroke Study. Stroke 27: 825-832
13. Karvonen M, Tuomilehto J, Libman I, LaPorte R (1993) A review of the recent epidemiological data on the worldwide incidence of type 1 (insulin-dependent) diabetes mellitus. World Health Organization DIAMOND Project Group. Diabetologia 36: 883-892

14. Lounamaa R (1993) Mortality in Finnish patients with insulin-dependent diabetes mellitus [Academic dissertation]. Social Insurance Institution, Helsinki

15. Salomaa V, Arstila M, Kaarsalo E et al. (1992) Trends in the incidence of and mortality from coronary heart disease in Finland, 1983-1988. Am J Epidemiol 136: 1303-1315

16. Diabetes Epidemiology Research International Mortality Study Group (1991) International evaluation of cause specific mortality. Diabetes Care 14: 55-60

17. Diabetes Epidemiology Research International Mortality Study Group (1991) Major cross-country differences in risk of dying for people with IDDM. Diabetes Care 14: 49-54

18. Tuomilehto J, Rewers M, Reunanen A et al. (1991) Increasing trend in type 1 (insulin-dependent) diabetes mellitus in childhood in Finland. Analysis of age, calendar time and birth cohort effects during 1965 to 1984 . Diabetologia 34: $282-287$

19. Åkerblom HK, Reunanen A (1985) The epidemiology of insulin-dependent diabetes mellitus (IDDM) in Finland and in northern Europe. Diabetes Care 8 [Suppl 1]: 10-16

20. Mähonen M, Salomaa V, Brommels M et al. (1997) The validity of hospital discharge register data on coronary heart disease in Finland. Eur J Epidemiol 13: 403-415

21. Heliövaara M, Reunanen A, Aromaa A, Knekt P, Aho K, Suhonen O (1984) Validity of hospital discharge data in a prospective epidemiological study on stroke and myocardial infarction. Acta Med Scand 216: 309-315

22. Tuomilehto J, Borch-Johnsen K, Molarius A et al. (1997) The unchanging incidence of hospitalization for diabetic nephropathy in a population-based cohort of IDDM patients in Finland. Diabetes Care 20: 1081-1086

23. Salomaa V, Miettinen H, Kuulasmaa K et al. (1996) Decline of coronary heart disease mortality in Finland during 1983 to 1992: roles of incidence, recurrence, and case-fatality. The FINMONICA MI Register Study. Circulation 94: 3130-3137

24. Tuomilehto J, Sarti C, Narva EV et al. (1992) The FINMONICA Stroke Register. Community-based stroke registration and analysis of stroke incidence in Finland, 1983-1985. Am J Epidemiol 135: 1259-1270

25. DERI Mortality Study Group (1991) Sex differences in the mortality associated with insulin-dependent diabetes mellitus in four countries. Am J Epidemiol 133: 577-584

26. Tuomilehto J, Rastenyte D, Jousilahti P, Sarti C, Vartiainen E (1996) Diabetes mellitus as a risk factor for death from stroke: prospective study of the middle-aged Finnish population. Stroke 27: 210-215

27. Parving HH, Andersen AR, Smidt UM, Christiansen JS, Oxenboll B, Svendsen PA (1983) Diabetic nephropathy and arterial hypertension. The effect of antihypertensive treatment. Diabetes 32 [Suppl 2]: 83-87

28. Feldt-Rasmussen B, Borch-Johnsen K, Mathiesen ER (1985) Hypertension in diabetes as related to nephropathy. Early blood pressure changes. Hypertension 7: II18-II20

29. Jensen T, Borch-Johnsen K, Deckert T (1987) Changes in blood pressure and renal function in patients with type I (insulin-dependent) diabetes mellitus prior to clinical diabetic nephropathy. Diabetes Research 4: 159-162

30. Christiansen JS (1978) Cigarette smoking and prevalence of microangiopathy in juvenile-onset insulin-dependent diabetes mellitus. Diabetes Care 1: 146-149 
31. Feldt-Rasmussen B (1986) Increased transcapillary escape rate of albumin in type 1 (insulin-dependent) diabetic patients with microalbuminuria. Diabetologia 29: 282-286

32. Valdorf-Hansen F (1967) Coagulability in diabetics. Acta Med Scand [Suppl] 476: 147-157

33. Jensen T, Stender S, Deckert T (1988) Abnormalities in plasma concentrations of lipoproteins and fibrinogen in type 1 (insulin-dependent) diabetic patients with increased urinary albumin excretion. Diabetologia 31: 142-145

34. Jones SL, Close CF, Mattock MB, Jarrett RJ, Keen H, Viberti GC (1989) Plasma lipid and coagulation factor concentrations in insulin dependent diabetics with microalbuminuria. Br Med J 298: 487-490

35. Nikkilä EA, Hormila P (1978) Serum lipids and lipoproteins in insulin-treated diabetes. Demonstration of increased high density lipoprotein concentrations. Diabetes 27: 1078-1086

36. Mattock MB, Salter AM, Fuller JH (1982) High density lipoprotein subfractions in insulin-dependent and normal subjects. Atherosclerosis 45: 67-79

37. Kahri J, Groop PH, Viberti G, Elliott T, Taskinen MR (1993) Regulation of apolipoprotein A-I-containing lipoproteins in IDDM. Diabetes 42: 1281-1288
38. Miller GJ, Miller NE (1975) Plasma-high-density-lipoprotein concentration and development of ischaemic heartdisease. Lancet 1: 16-19

39. Salonen JT, Salonen R, Seppänen K, Rauramaa R, Tuomilehto J (1991) HDL, HDL2, and HDL3 subfractions, and the risk of acute myocardial infarction. A prospective population study in eastern Finnish men. Circulation 84: 129-139

40. Billman GE, Schwartz PJ, Stone HL (1982) Baroreceptor reflex control of heart rate: a predictor of sudden cardiac death. Circulation 66: 874-880

41. Kleiger RE, Miller JP, Bigger JT Jr, Moss AJ (1987) Decreased heart rate variability and its association with increased mortality after acute myocardial infarction. Am J Cardiol 59: 256-262

42. Billman GE, Schwartz PJ, Stone HL (1984) The effects of daily exercise on susceptibility to sudden cardiac death. Circulation 69: 1182-1189

43. Ewing DJ (1984) Cardiac autonomic neuropathy. In: Jarrett RJ (ed) Diabetes and heart disease. Elsevier, New York, pp 99-132

44. Mölgaard H, Sörensen KE, Bjerregaard P (1991) Circadian variation and influence of risk factors on heart rate variability in healthy subjects. Am J Cardiol 68: 777-784 